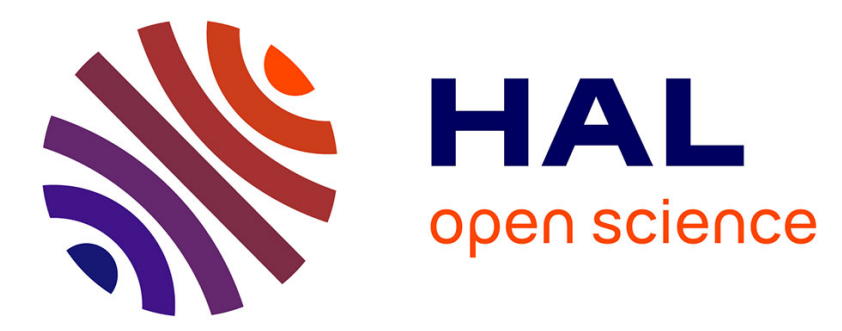

\title{
Prediction of symmetry during intermittent and annular horizontal two-phase flows
}

\author{
T. Layssac, C. Capo, S. Lips, A. W. Mauro, R. Revellin
}

\section{To cite this version:}

T. Layssac, C. Capo, S. Lips, A. W. Mauro, R. Revellin. Prediction of symmetry during intermittent and annular horizontal two-phase flows. International Journal of Multiphase Flow, 2017, 95, pp.91-100. 10.1016/j.ijmultiphaseflow.2017.05.010 . hal-01553471

\section{HAL Id: hal-01553471 \\ https://hal.science/hal-01553471}

Submitted on 20 Mar 2019

HAL is a multi-disciplinary open access archive for the deposit and dissemination of scientific research documents, whether they are published or not. The documents may come from teaching and research institutions in France or abroad, or from public or private research centers.
L'archive ouverte pluridisciplinaire HAL, est destinée au dépôt et à la diffusion de documents scientifiques de niveau recherche, publiés ou non, émanant des établissements d'enseignement et de recherche français ou étrangers, des laboratoires publics ou privés. 


\title{
Prediction of symmetry during intermittent and annular horizontal two-phase flows
}

\author{
Thibaut Layssace,", Claudia Capo², Stéphane Lips ${ }^{1}$, Alfonso William Mauro² and Rémi Revellin ${ }^{1}$ \\ ${ }^{1}$ Univ Lyon, CNRS, INSA-Lyon, CETHIL UMR5008, F-69621, Villeurbanne, France \\ thibaut.layssac@insa-lyon.fr
}

${ }^{2}$ Dipartimento di Ingegneria Industriale, Università degli Studi di Napoli “Federico II”, P.le V. Tecchio 80, 80125, Napoli, Italy

\begin{abstract}
An optical method is developed for a $2.95 \mathrm{~mm}$ inner diameter circular mini-channel to estimate liquid film thicknesses and then characterize symmetry of R-245fa horizontal two-phase flow. The greyscale pictures obtained with a high-speed camera are processed to determine the liquid-vapour interface positions for annular and intermittent flows. The experiments are thus performed for a large range of flow conditions. The saturation temperatures tested ranged from $20^{\circ} \mathrm{C}$ to $100^{\circ} \mathrm{C}$ in steps of $10^{\circ} \mathrm{C}$ and the mass velocities are $50,100,200,300$ and $400 \mathrm{~kg} \cdot \mathrm{m}^{-2} \cdot \mathrm{s}^{-1}$. A new parameter ranging from 0 to 1 , the symmetry, is defined to account for the level of non uniformity of liquid distribution around the tube perimeter. New experimental data are presented (270 data points), that cover a range of symmetry parameter from 0.35 to 1.00 . These data are merged with those available in the literature (406 data points with symmetry parameter from 0.71 to 1.00 ). A sensitivity analysis of the dimensionless numbers of major influence is run and a new correlation is proposed, that enables to predict over $90 \%$ of the data points in an error bandwidth of $10 \%$. This correlation is suggested in the determination of an axisymmetric criterion.
\end{abstract}

Keywords: symmetry, asymmetry, film thickness, intermittent flow, annular flow, correlation

\section{Introduction}

Two-phase flows occur in a large range of industrial applications corresponding to various domains as chemical processing, petrochemical transportation, evaporators and condensers or steam generators in nuclear plants. The two-phase flow geometry affects the performance of these applications and is strongly linked to inertia, buoyancy, surface tension or shear stresses. All these forces are influenced by the two-phase flow parameters such as vapor quality, mass velocity or temperature but also by the orientation of the flow. In the case of a vertical configuration, gravity being in the two-phase flow direction, the flow is axisymmetric. In the case of a horizontal configuration, gravity being orthogonal to the direction of the flow, the vapor phase tends to move to the top of the channel. Thus, knowledge of geometrical aspects, such as the distribution of liquid around the circumference of the tube, is essential in the understanding of horizontal two-phase flow behaviours. Studies on film thickness distribution can be motivated by the understanding of heat transfer mechanisms or gas-liquid interface kinematic behaviours such as pumping waves. Different interpretations of the phenomena 
were proposed to describe the circumferential film distribution. Among them exists the hypothesis of a secondary flow in the gas core, draining liquid to the top of the internal tube wall, promoted by Pletcher and McManus (1965) and then elevated by Laurinat et al. (1984) and Lin et al. (1986). Studies on wave behaviours were also led by Butterworth (1972), Fukano and Ousaka (1989) and Jayanti et al. (1990). The description of hydraulic mechanisms, such as pumping waves, aimed to explain the geometrical configuration, and was related to the evolution of asymmetry with flow parameters.

More recently, a number of studies dealing with asymmetry were carried out, using different formulations of vapour Froude number, as a tool to correlate data. For instance, Williams et al. (1996), Hurlburt and Newell (1997), Schubring and Shedd (2009) and Cioncolini and Thome (2013) proposed various correlations. Williams et al. (1996) observed the evolution of the two-phase flow asymmetry as a function of gas velocity. Most asymmetric flows occur for low gas velocities and the flow tends to be symmetric for high gas velocity. Hurlburt and Newell (1997) proposed a liquid film asymmetry correlation and a liquid film thickness distribution model based on stratified-annular, asymmetric and symmetric annular flows. One of the purposes of Schubring and Shedd (2009) study was to model the base film thickness and to quantitatively link this parameter with the symmetry by the medium of a modified vapour Froude number. This study was led with very slightly stratified two-phase flows. Cioncolini and Thome (2013) considered a new prediction method of asymmetry and developed a flow-pattern map which enables to discriminate asymmetric and symmetric twophase flows. Hurlburt and Newell (1997), Schubring and Shedd (2009) and Cioncolini and Thome (2013) all described the asymmetry by means of eccentricity which considers a ratio of film thicknesses along the tube circumference.

More recently, Donniacuo et al. (2015) introduced a new definition of eccentricity which was linearly linked to the position of vapour core centre and consequently was based on the difference between the top and the bottom film thicknesses. The eccentricity was qualitatively linked with the flow parameters and commented not only with vapour Froude number but also with Bond number. This last dimensionless number enabled to show the effect of capillary forces on asymmetry, forces which occur for lower diameters than in previous studies.

In the present study, the optical method and the numerical method are presented. They derived from that of Donniacuo et al. (2015) and were used and adapted to build the experimental database. This adaptation enables to study horizontal annular and intermittent flows. In the second part, a new symmetry parameter is presented. It is based on vapour core centre which is a way to describe the qualitative behaviour of the flow asymmetry in the present case. Then, the present database is described, analyzed and a comparison among previous asymmetry correlations against the present database is carried out with statistical indicators. Observations on these correlations accuracy lead to the presentation of a new correlation, preceded by the description of the method used for its derivation. This new prediction method was compared to both present databases and an existing one from literature. 


\section{Presentation of the experimental database}

\subsection{Description of the experimental test bench}

The test bench, designed and previously described by Charnay et al. (2014), enables to set the pressure, the enthalpy, the heat flux density and the mass velocity conditions at the test section inlet. The test section presented in figure 1 consists in three parts, consisting of a $2000 \mathrm{~mm}$ spirally shaped preheater, a $185 \mathrm{~mm}$ horizontal evaporator and a $200 \mathrm{~mm}$ glass visualization tube. The preheater ensures the control of the vapour quality at the test section inlet. The vapour quality and the saturation pressure of the glass tube are evaluated at the outlet of the evaporator by assuming the pressure drop and the heat losses as negligible. The test section is presented in figure 1 .

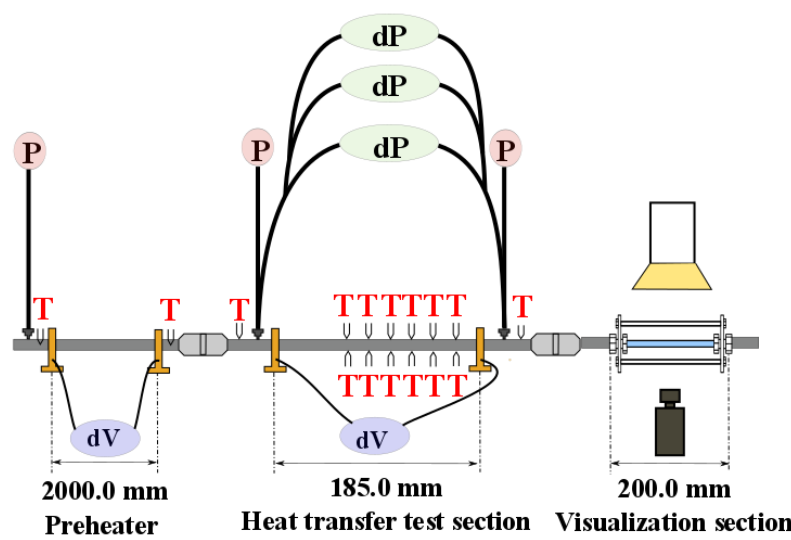

Figure 1: Schematic of the test section (Charnay et al., 2014)

An optical measurement technique was developed to measure the two-phase flow liquid film thickness in the $2.95 \mathrm{~mm}$ inner diameter glass tube (Donniacuo et al., 2015). A high-speed camera system and a light behind the tube enable the visualization of the flow by recording frame sequences. The camera enables to get the local liquid film thickness with a mean resolution of 200 pixels. $\mathrm{mm}^{-1}$.

In each condition of temperature, mass velocity and vapour quality, 4 series of 1363 frames are taken, corresponding to a total time of $2.7 \mathrm{~s}$. The frame size is of $1024 \times 1024$ pixels and each pixel has a greyscale value ranging from 0 to 255 .

A Matlab program was conceived and enables to determine the local film thickness for annular flows (Donniacuo et al., 2015). This method based on greyscale analysis was improved to be applied to intermittent flows where the single-phase profiles have to be detected. This method also enables to sensibly reduce the uncertainty on the interface detection for the annular parts. Since the direct measurement suffers from the refraction effects through the glass of the tube, a correction factor has been introduced: 
$t_{\text {real }}=\frac{t_{a p p}}{S c \times E F}$

with $t_{a p p}$ the apparent film thickness, $t_{\text {real }}$ the real film thickness, $E F$ the enlargement factor due to optical deformation, and $S c$ the scaling factor.

The uncertainty on the apparent film thickness is \pm 2 pixels. At each operating condition, the top and bottom liquid film thicknesses were calculated as the average value from 5452 recorded frames. The uncertainty of each measurement is calculated, taking into account several sources of uncertainty: the inner and outer diameter dimensions, the variation of liquid R-245fa refractive index with the temperature and the limitations of image resolution due to finite pixel dimensions. The combined uncertainty for film thickness measurement was evaluated. In the present study, the uncertainty on the apparent film thickness is equal to 2 pixels, the enlargement factor uncertainty is 0.047 and the scaling conversion uncertainty is $0.84 \mathrm{~mm} /$ pixel. All these uncertainties were measured for an outer apparent diameter of 909 pixels. As a conclusion, the uncertainty on the estimation of the real top film thickness ranges between $0.02 \mathrm{~mm}$ and $0.03 \mathrm{~mm}$ depending on the experimental conditions.

\subsection{New experimental data points}

The experiments are performed with R-245fa for mass velocities equal to 50, 100, 200, 300 and $400 \mathrm{~kg} \cdot \mathrm{m}^{-2} \cdot \mathrm{s}^{-1}$, and for saturation temperatures ranging from $20^{\circ} \mathrm{C}$ to $100^{\circ} \mathrm{C}$ in steps of $10^{\circ} \mathrm{C}$. This range of temperature enables to have important variations of the thermophysical properties as densities, viscosities and surface tension. These variations of saturation conditions change the relative effect of the difference forces acting on the two-phase equilibrium. It strongly affects the liquid-vapour interface and then on the flow pattern. The large vapour quality range enables to get the transition between intermittent and annular flow.

The variations of the two-phase flow geometry can be first characterized by considering the evolution of the film thicknesses at the top and at the bottom sides of the tube for various saturation temperatures $T_{\text {sat }}$ and a given mass velocity $G$ of $200 \mathrm{~kg} \cdot \mathrm{m}^{-2} \cdot \mathrm{s}^{-1}$ (figure 2). It is shown that both film thicknesses decrease with vapour quality. The top film thickness is about ten times lower than the bottom film thickness. Contrarily to the top film thickness, the bottom film thickness clearly increases with the saturation temperature.

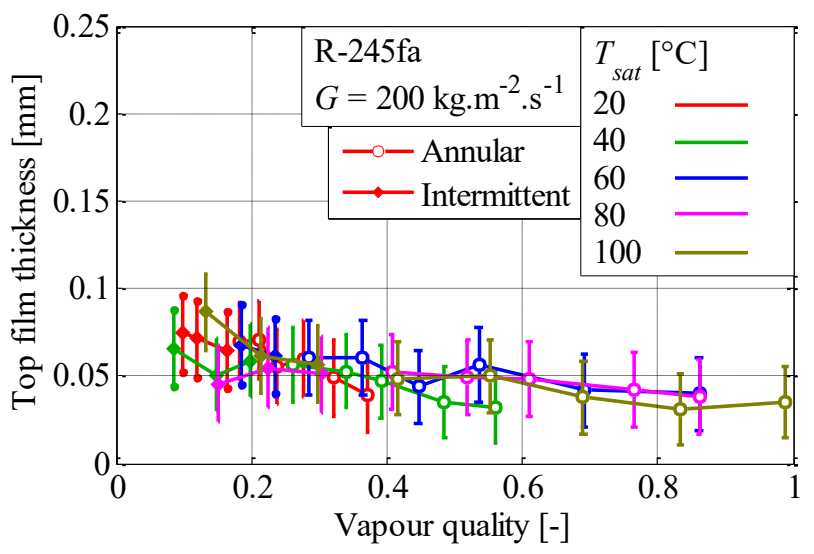


a) Top film thickness

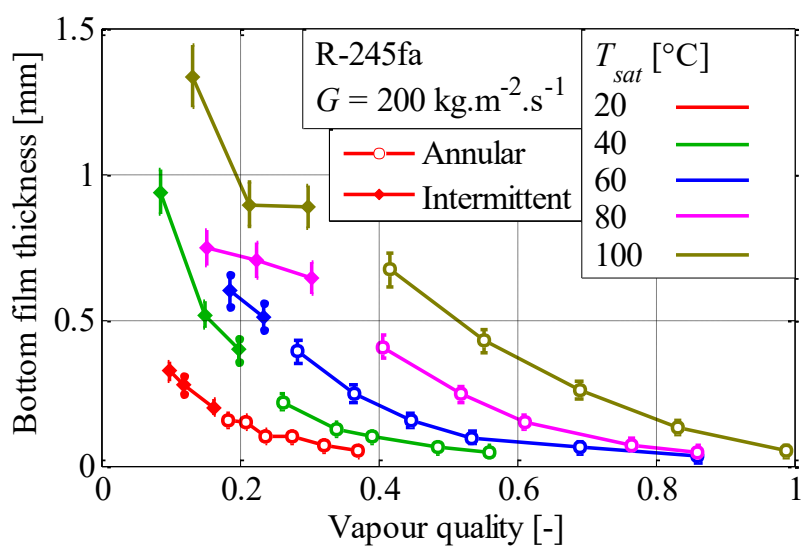

b) Bottom film thickness

Figure 2: Evolution of the top and bottom film thicknesses for various saturation temperatures

The evolution of the film thicknesses affects the symmetry of the flow. It is possible to evaluate the evolution of the two-phase flow symmetry by considering the parameter $s$ which is defined as:

$s=\frac{d_{t o p}}{r}$

with $s$ the symmetry parameter, $d_{t o p}$ the distance between the vapour core centre and the top glass-liquid interface and $r$ the internal radius as shown in figure 3.

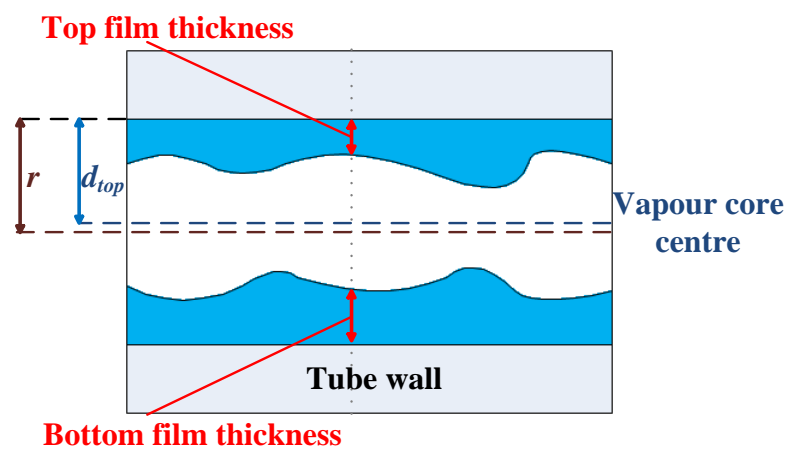

Figure 3: Position of the vapour core centre for a horizontal two-phase flow

This parameter definition is strictly equivalent to:

$s=1-\frac{t_{\text {bottom }}-t_{\text {top }}}{d}$

where $d$ is the internal diameter, $t_{\text {bottom }}$ and $t_{\text {top }}$ are respectively the bottom and the top film thicknesses. In practice, the symmetry parameter $s$ is evaluated with this previous equation. This definition admits two limit cases. When the flow is centred, $t_{\text {bottom }}=t_{\text {top }}$ and $s=1$. On the contrary, when a bubble appears at the top glass-liquid interface, $t_{\text {bottom }} \rightarrow d$ and $t_{\text {top }} \rightarrow 0$, which corresponds to $s=0$. In the case of an intermittent flow, the symmetry is calculated considering both top and bottom mean film thicknesses on two-phase flow parts. Thus, the liquid plugs are not considered in the calculation of symmetry. 
Taking into account the propagation of uncertainties of the both film thicknesses and the internal diameter measurements, the mean absolute uncertainty of the symmetry parameter is equal to 0.025 and its maximum value is equal to 0.055 .

Figure 4 presents the evolution of symmetry $s$ with vapour quality for a mass velocity of $200 \mathrm{~kg} \cdot \mathrm{m}^{-2} \cdot \mathrm{s}^{-1}$ and for temperatures of $20,40,60,60,80$ and $100^{\circ} \mathrm{C}$. First, when vapour quality gets higher, two-phase flow is more centred, tending to the limit case of a centred vapour flow for $s=1$. Secondly, the flow tends to be symmetric when the temperature decreases.

Figure 5 deals with the symmetry behaviour for different mass velocities of $50,100,200,300$ and $400 \mathrm{~kg} . \mathrm{m}^{-2} . \mathrm{s}^{-}$ ${ }^{1}$ and a fixed temperature of $60^{\circ} \mathrm{C}$. It shows that the flow symmetry increases with mass velocity.

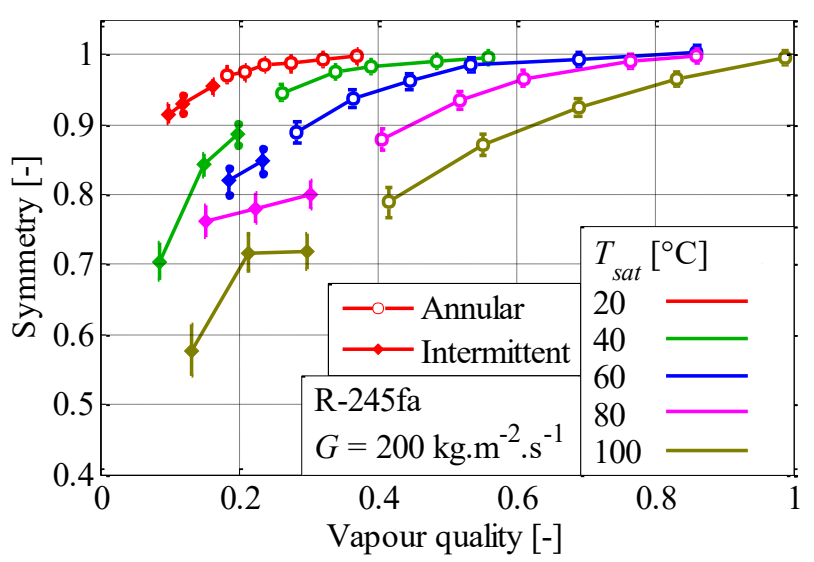

Figure 4: Influence of the saturation temperature on symmetry for mass velocity of $200 \mathrm{~kg} \cdot \mathrm{m}^{-2} \cdot \mathrm{s}^{-1}$

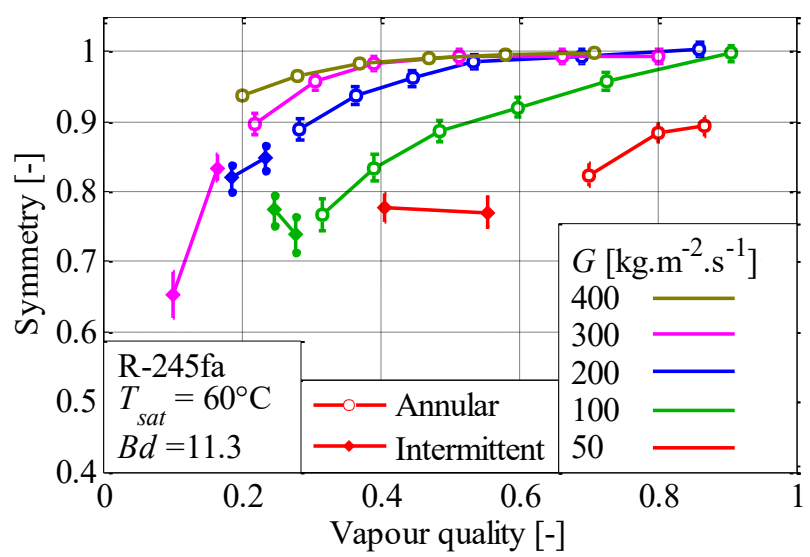

Figure 5: Influence of the mass velocity on symmetry for a saturation temperature of $60^{\circ} \mathrm{C}$

In the present study, the evolution of the symmetry is considered to be affected by three main kinds of force which are inertia, gravity, and surface tension forces. To evaluate the relative effects of these four forces in both phases, the Bond number and the vapour Froude number are considered. The present definition of the vapour Froude number was proposed by Cioncolini and Thome (2013). It compares the buoyancy effects with the vapour phase inertia. 
$B d=\frac{\text { gravity forces }}{\text { surface tension forces }}=\frac{g\left(\rho_{l}-\rho_{v}\right) d^{2}}{\sigma}$

$F r_{v}=\frac{\text { inertia forces }}{\text { gravity forces }}=\frac{\frac{x G}{\sqrt{\rho_{v}}}}{\sqrt{g\left(\rho_{l}-\rho_{v}\right) d}}$

According to equation (5), when the mass velocity or the vapour quality increases, the vapour Froude number increases. It corresponds to an increasing relative effect of inertia compared to buoyancy forces. Thus, buoyancy effects stratifying the flow, the symmetry parameter decreases. This number decreases with diameter which is consistent with the fact that two-phase flows are more stratified for higher internal diameter.

When temperature increases, the difference between vapour and liquid densities decreases and surface tension also decreases. However, the difference decreases slower than the surface tension term. Relative effect of gravity forces above capillary forces increases and the Bond number increases according to equation (4). The flow is less symmetric.

The evolution of the two-phase flow symmetry $s$ with the Bond number and the vapour Froude number is qualitatively summarized in table 1 .

Table 1: Evolution of the symmetry with dimensionless numbers

\begin{tabular}{llll}
\hline $\mathrm{Bd}$ & $\nearrow$ & $s$ & $\searrow$ \\
$\mathrm{Fr}_{\mathrm{v}}$ & $\nearrow$ & $s$ & $\nearrow$
\end{tabular}

The experiments performed with R-245fa saturated flow and the optical diagnostic enabled to follow the evolution of the two-phase flow geometry for a large range of flow patterns, i.e. intermittent and annular flows. The introduction of the symmetry $s$ coupled with dimensionless numbers such as the vapour Froude number and the Bond number led to a first description of the evolution of two-phase flow symmetry with the flow parameters.

\section{Comparison with existing prediction methods}

The evolution of the dimensionless numbers as the vapour Froude and Bond numbers enables a first qualitative analysis of the symmetry dependence to flow parameters. Cioncolini and Thome (2013), Schubring and Shedd (2009) and Hurlburt and Newell (1997) proposed to predict quantitatively this evolution mainly for slightly asymmetric air-water flows. In the works of Cioncolini and Thome (2013) and Schubring and Shedd (2009), the eccentricity, which describes the flow asymmetry, is defined as the ratio between top film thickness and bottom film thickness.

$e c c_{\text {ratio }}=\frac{t_{\text {top }}}{t_{\text {bottom }}}$

where $t_{\text {top }}$ and $t_{\text {bottom }}$ are respectively the top and bottom film thicknesses. This definition gets the same limits as the symmetry parameter for fully stratified and centred flows. 
The expression proposed by Cioncolini and Thome (2013) to evaluate the eccentricity ecc ratio is:

$e c c_{\text {ratio }}=\frac{0.0789 F r_{v}^{1.90}}{1+0.0789 F r_{v}^{1.90}} ; F r_{v}>1$

Schubring and Shedd (2009) proposed the following equation to predict asymmetry:

$e c c_{\text {ratio }}=1-\exp \left(-0.63 F r_{s s}\right)$

with $F r_{s S}=\frac{x G}{\rho_{l}\left(g t_{\text {mean }}\right)^{0.5}}$

$t_{\text {mean }}$ is the mean film thickness calculated with the following empirical correlation:

$\frac{t_{\text {mean }}}{d}=\frac{4.7}{x}\left(\frac{\rho_{v}}{\rho_{l}}\right)^{\frac{1}{3}}\left(\frac{G d}{\mu_{l}}\right)^{-\frac{2}{3}}$

Hurlburt and Newell (1997) proposed to predict stratification by introducing the mean film thickness on the tube circumference as follows and introduced the following ratio:

$e c c_{\text {mean }}=\frac{t_{\text {mean }}}{t_{\text {bottom }}}$

This evaluation of two-phase flow asymmetry leads to the following expression:

$e c c_{\text {mean }}=0.2+0.7\left(1-e^{-\frac{1}{75}\left(\left(\frac{x}{1-x}\right)^{0.5} F r_{h n}-20\right)}\right)$

valid for $\left(\frac{x}{1-x}\right)^{0.5} F r_{h n}>20$ and with $F r_{h n}=\frac{x G}{\rho_{v}(g d)^{0.5}}$

These three studies were led with a large number of experimental or bibliographic data points. In the case of Schubring and Shedd (2009), the correlation was obtained considering their own experimental data points only while Cioncolini and Thome (2013) optimization was enabled thanks to a bibliographic study. The film thicknesses around the tube circumference were measured for a large range of conditions, especially considering the tube size. Table 2 presents the ranges of internal diameters, gas quality, symmetry $s$, vapour Froude number, Bond number and number of points considered in each mentioned study database and in the current experimental study database.

Table 2: Bibliography and present data ranges

\begin{tabular}{cccccccc}
\hline & Fluid & $d[\mathrm{~mm}]$ & $x[-]$ & $s[-]$ & Points [-] & $\mathrm{Bd} \mathrm{[-]}$ & $\mathrm{Fr}_{\mathrm{v}}[-]$ \\
\hline $\begin{array}{c}\text { Hurlburt and Newell } \\
(1997)\end{array}$ & Air-water & $23 \rightarrow 95$ & $0.07 \rightarrow 0.96$ & $0.82 \rightarrow 1.00$ & 105 & $87 \rightarrow 347$ & $0.7 \rightarrow 9.8$ \\
$\begin{array}{c}\text { Schubring and Shedd } \\
(2009)\end{array}$ & Air-water & $8.8 \rightarrow 26.3$ & $0.11 \rightarrow 0.76$ & $0.99 \rightarrow 1.00$ & 206 & $10 \rightarrow 93$ & $2.2 \rightarrow 9.7$ \\
$\begin{array}{c}\text { Cioncolini and Thome } \\
(2013)\end{array}$ & Air-water & $12 \rightarrow 95$ & $0.03 \rightarrow 0.96$ & $0.82 \rightarrow 1.00$ & 182 & $8.9 \rightarrow 1200$ & $0.7 \rightarrow 9.8$ \\
$\begin{array}{c}\text { Masala et al. } \\
(2007)\end{array}$ & Air-water & 21 & $0.04 \rightarrow 0.21$ & $0.84 \rightarrow 0.89$ & 6 & 59 & 0.3 \\
\hline
\end{tabular}




\begin{tabular}{cccccccc}
\hline $\begin{array}{c}\text { Ong and Thome } \\
(2011)\end{array}$ & R-236fa & 1.03 & $0.16 \rightarrow 0.69$ & $0.71 \rightarrow 1.00$ & 12 & $1.1 \rightarrow 1.5$ & $1.2 \rightarrow 7.9$ \\
$\begin{array}{c}\text { R-245fa } \\
\begin{array}{c}\text { Donniacuo et al. } \\
(2015)\end{array}\end{array}$ & R-245fa & 2.95 & $0.1 \rightarrow 0.82$ & $0.83 \rightarrow 1.00$ & 41 & $9.2 \rightarrow 18.6$ & $1.8 \rightarrow 11$ \\
$\begin{array}{c}\text { Present experiments } \\
\text { Total }\end{array}$ & R-245fa & 2.95 & $0.06 \rightarrow 0.99$ & $0.35 \rightarrow 1.00$ & 229 & $7.7 \rightarrow 19.1$ & $0.4 \rightarrow 8.3$ \\
\hline & & $1.03 \rightarrow 95$ & $0.03 \rightarrow 1.00$ & $0.35 \rightarrow 1.00$ & 676 & $1.1 \rightarrow 1200$ & $0.3 \rightarrow 11$ \\
\hline
\end{tabular}

In the present study, 63 measurements were made with symmetry $s$ lower than 0.82 which is the lowest limit of the other studies dedicated to the determination of an eccentricity correlation. It represents $8 \%$ of the total database ( $28 \%$ of the present database). Geometrically, this means the two-phase flows which were studied on the present test bench were more asymmetric than in the past studies which dealt with annular flows only. In the present case, it was done by considering intermittent flows which are more asymmetric than annular flows. The symmetry predictions of the three mentioned correlations are evaluated on the total database gathering 676 points. This evaluation is qualitatively enabled by considering the distribution of measured symmetry as a function of the predicted symmetry for each model. The evaluation of the mean film thickness is done considering all the circumferential measurement positions for each study.

In figure 6, the data points that do not respect the criterion given for using correlations of Hurlburt and Newell (1997) and Cioncolini and Thome (2013) are in light grey. All these points are not considered in the calculation of statistical indicators. The quantitative comparison is made by calculating the percentage of measured eccentricity whose relative error is under $50 \%$. Furthermore, the relative error can be evaluated by introducing the mean absolute percentage error:

$M A P E=\frac{1}{N} \sum_{i=1}^{N}\left|\frac{e c c_{\text {pred }, i}-e c c_{\text {meas }, i}}{e c c_{\text {meas }, \mathrm{i}}}\right|$

where $N$ is the sample size, $e c c_{\text {pred }}$ the predicted eccentricity and $e c c_{\text {meas }}$ the measured eccentricity.

To evaluate if the measured eccentricities are globally underestimated or overestimated, the mean percentage error is an appropriate statistical indicator:

$M P E=\frac{1}{N} \sum_{i=1}^{N} \frac{e c c_{\text {pred }, i}-e c c_{\text {meas }, i}}{e c c_{\text {meas }, i}}$

The Standard deviation of Absolute Percentage Error is introduced to give information about the distribution of the relative errors. The lower this indicator is, the less dispersed the data prediction is. Consequently, when this indicator is low, all the data points are predicted with the same accuracy.

$S A P E=\sqrt{\frac{1}{N} \sum_{i=1}^{N}\left(\left|\frac{e c c_{\text {pred }, i}-e c c_{\text {meas }, i}}{e c c_{\text {meas }, \mathrm{i}}}\right|-M A P E\right)^{2}}$ 


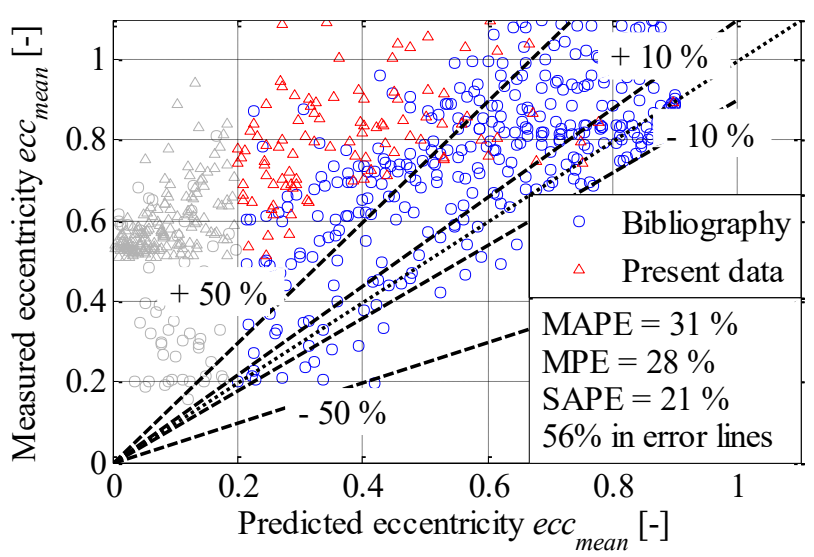

a) Hurlburt and Newell (1997)

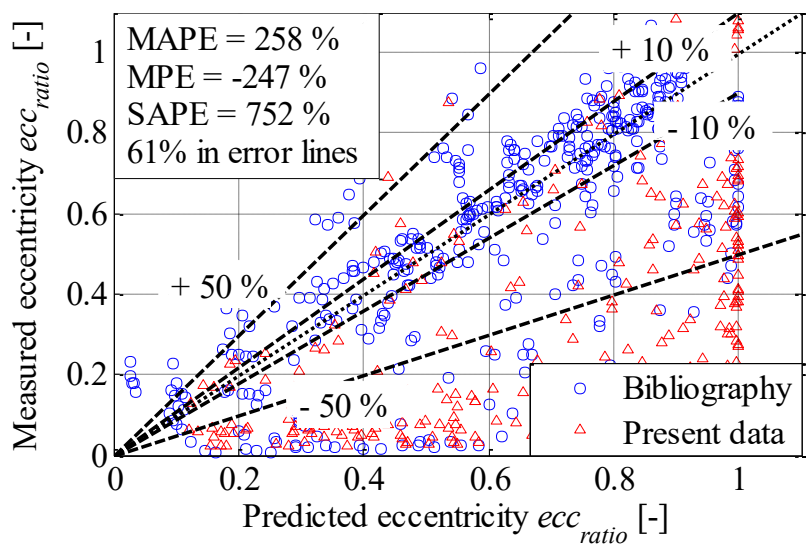

b) Schubring and Shedd (2009)

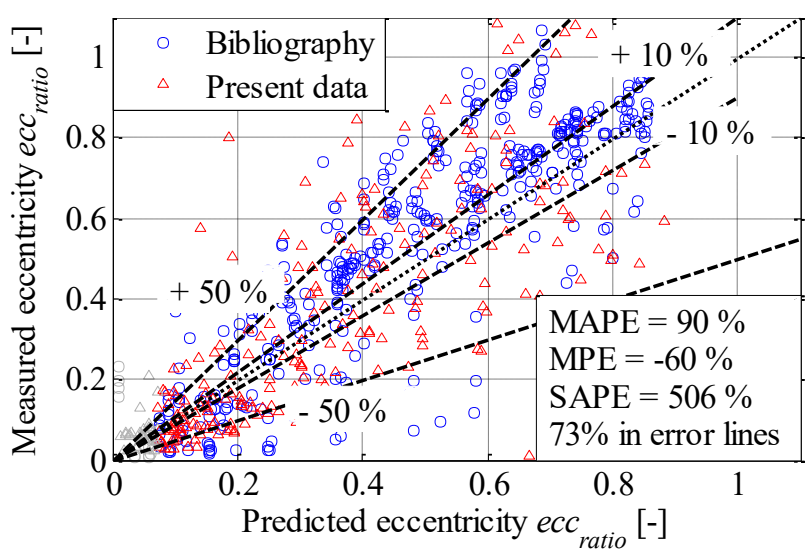

c) Cioncolini and Thome (2013)

Figure 6: Donniacuo et al. (2015) and present measurements data compared to the correlations of a) Hurlburt and Newell (1997), b) Schubring and Shedd (2009), c) Cioncolini and Thome (2013)

Hurlburt and Newell correlation (1997) underestimates the eccentricity, which is statistically indicated by a positive mean percentage error. In the opposite way, the correlations of Cioncolini and Thome (2013) and Schubring and Shedd (2009) overestimate the measured eccentricity. The high mean percentage error from Schubring and Shedd (2009) correlation is directly linked to the symmetry $s$ range considered in their database. All their data thickness measurements were made with a symmetry $s$ higher than 0.99 . This study reports an 
asymptotic behaviour which corresponds to only a little part of the total database. The standard deviation of the absolute percentage for the data predictions of Cioncolini and Thome (2013) and Schubring and Shedd (2009) indicates these correlations predict the data with a high dispersion. Contrarily, Hurlburt and Newell correlation (1997) predicts all the data with the same accuracy.

Globally, the correlation proposed by Hurlburt and Newell (1997) is the most accurate on the total database in terms of mean absolute percentage error and mean percentage error while Cioncolini and Thome correlation (2013) captures the highest amount of data points with a relative error of 50\%. However, the accuracy of the symmetry quantitative prediction still needs to be improved and a new correlation is then proposed.

\section{Development of a new correlation}

\subsection{Analysis of the correlation}

The qualitative description of symmetry dependence on flow parameters showed that two dimensionless numbers can be used to describe the flow conditions: the vapour Froude number and the Bond number, taking into account inertia, buoyancy and capillary effects. The observed evolution of symmetry with these dimensionless numbers suggests correlating database measurements of symmetry parameter $s$ with dimensionless numbers. The limit cases of 0 for bubble disappearance at the top of the tube and 1 for a centred flow have to be respected. The monotonic evolution of symmetry with the vapour Froude number and the Bond number also suggests choosing a monotonic function. The function used for respecting these mathematical restrictions can then be constructed with the following form:

$$
s=\frac{1}{1+h \prod_{i=1}^{N} P_{i}^{\alpha_{i}}}
$$

where $h$ is the multiplying factor $(h>0), P_{i}$ is the ith dimensionless physical parameter, $\alpha_{i}$ the ith exponent to be determined.

All the coefficients of the correlation are obtained by means of a nonlinear method based on the minimization of the Mean Squared Error defined as:

$$
M S E=\frac{1}{N} \sum_{i=1}^{N}\left(s_{\text {meas }, i}-s_{\text {pred }, i}\right)^{2}
$$

An optimization is then led considering both phases viscosity, density, and quality, surface tension, mass velocity and diameter. This first optimization enables to obtain a distribution of the symmetry parameter $s$ for the present database which is presented in figure 7. 


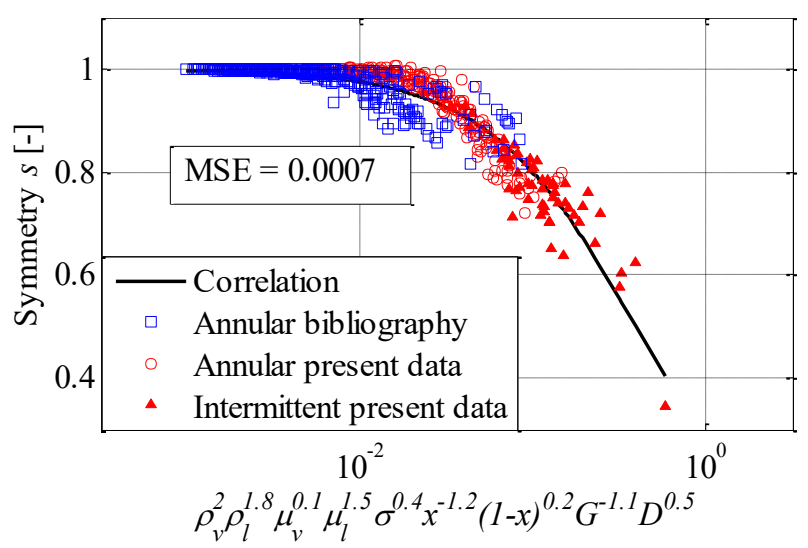

Figure 7: Base parameters correlation

The set of exponents presented on figure 7 enables a good representation of the database. However, the optimization algorithm implies to determine 10 parameters ( 9 exponents and the multiplying factor $h$ ) which can affect the numerical robustness of the method. In order to quantify this robustness, a sensitivity analysis of the quality of the optimization to each exponent is led.

In practice, when the algorithm is run, it searches for the values of exponents that minimize the Mean Squared Error. The MSE can be more or less affected by each exponent variation during this research of a global minimum. Consequently, the sensitivity study consists into imposing a given value of the exponent whose impact is tested, and running the optimization algorithm with the other exponents. If the imposed exponent differs from the optimum one, the MSE is higher than that of the global minimum previously found. The evolution of the MSE as a function of the fixed exponent is directly an image of the impact of this exponent on the global optimization. If the MSE is strongly affected by the fixed exponent, it means that the concerned physical parameter has a strong influence on the flow symmetry and that its effect cannot be compensated by the other parameters. On the contrary, if the MSE is slightly affected by the exponent value, it means that either the physical parameter has a low effect on the flow symmetry or that its effect is numerically compensated by the other parameters.

The evolution of the MSE as a function of the imposed exponent for each parameter is presented in Figure 8. It shows the MSE is more affected by vapour quality, liquid quality, mass velocity and diameter than by the thermophysical parameters. As a consequence, the values on the exponents linked to these thermophysical parameters and determined by the algorithm have a low physical meaning. For instance, the exponent on the vapour viscosity could range from -1 to 1 without affecting the overall quality of the correlation. 


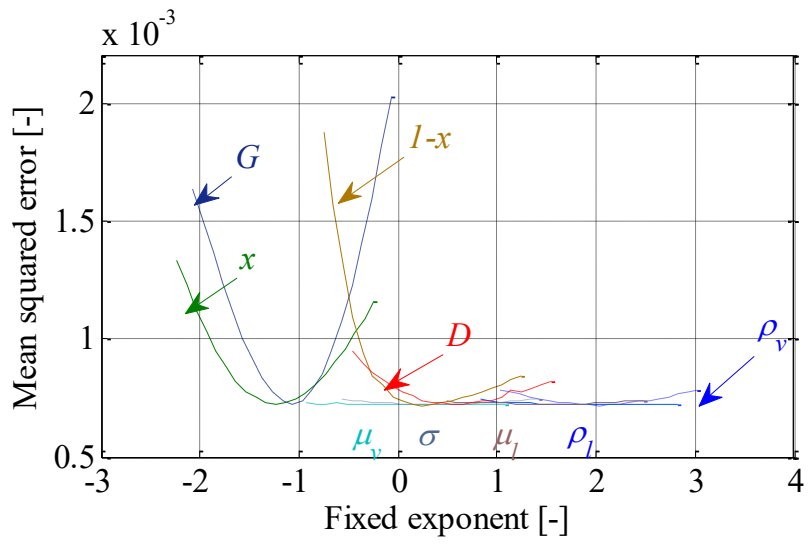

Figure 8: Evolution of the Mean Squared Error with the imposed exponents

The difference between the two groups of exponents suggests the method of optimization is more sensitive to the exponent value of vapour quality, liquid quality, mass velocity and diameter. This observation can be enlightened by the consideration of statistical correlations in the present database. The analysis of statistical correlations of the database enables to figure out the linear dependences between all the flow parameters. This analysis is made by means of the calculation of the correlation coefficient for each pair of flow parameters $\left(P_{i}\right.$, $\left.P_{j}\right)$ :

$$
\operatorname{Corr}\left(P_{i}, P_{j}\right)=\frac{E\left(P_{i} P_{j}\right)-E\left(P_{i}\right) E\left(P_{j}\right)}{\sigma_{P_{i}} \sigma_{P_{j}}}
$$

with $E(X)$ the mathematical expectation, $\sigma_{X}$ the standard deviation of the variable $X$.

Coefficient correlation is equal to 1 when the two flow parameters are linearly dependent, 0 when they are linearly independent. For instance, in the present database, surface tension is strongly correlated to liquid viscosity while it is very slightly correlated to mass velocity. This analysis enables to have an image of the robustness of the present database and to detect potential statistical biases. The absolute value of each correlation coefficient is presented in Table 3 .

Table 3: Correlation coefficients of the present database flow parameters

\begin{tabular}{cccccccc}
\hline$\left|\operatorname{Corr}\left(P_{i}, P_{j}\right)\right|$ & $\rho_{v}$ & $\rho_{l}$ & $\mu_{v}$ & $\mu_{l}$ & $\sigma$ & $x$ & $G$ \\
\cline { 2 - 8 } & 0.49 & 0.61 & 0.65 & 0.70 & 0.67 & 0.09 & 0.21 \\
$G$ & 0.05 & 0.10 & 0.08 & 0.06 & 0.05 & 0.26 & \\
$x$ & 0.22 & 0.06 & 0.11 & 0.16 & 0.16 & & \\
$\sigma$ & 0.78 & 0.89 & 0.96 & 0.99 & & & \\
$\mu_{l}$ & 0.82 & 0.85 & 0.94 & & & &
\end{tabular}




\begin{tabular}{l|ll}
$\mu_{v}$ & 0.60 & 0.98 \\
$\rho_{l}$ & 0.42 & \\
\hline
\end{tabular}

Correlation coefficients are observed to be globally higher between all thermophysical parameters. This linear dependence is explained by the fact all these parameters are fixed by temperature and pressure. Since these parameters are strongly correlated, their exponents may balance out each other. It thus increases the uncertainty on their estimation. The correlation between the diameter and the thermophysical parameters is explained by the fact that the experimental database was made with a unique diameter for a large range of temperatures while the bibliography data was mainly obtained with several diameters and with ambient temperature. For instance, the present experimental database considers one diameter of $3 \mathrm{~mm}$ with temperature ranging from $20^{\circ} \mathrm{C}$ from $100^{\circ} \mathrm{C}$ while Cioncolini and Thome database is constituted of experimental data made with ambient temperature and diameters ranging from $12 \mathrm{~mm}$ to $95 \mathrm{~mm}$. This statistical bias artificially links the diameter to the thermophysical parameters. To improve the quality of the database, and thus the robustness of the correlation, more experimental data would be required to determine more accurately the effect of each parameter on the flow symmetry.

As a conclusion, this first set of exponents enables to compare the relative effect of each flow parameter on the flow symmetry and to verify the consistency between the symmetry dependence and the signs of these exponents. However, this kind of correlation is not dimensionally consistent. All these flow parameters have to be grouped together in dimensionless numbers and the choice of these grouping is crucial in the accuracy of final correlation.

\subsection{Development of the correlation with dimensionless numbers}

The previous observations on the statistical correlations and the evolution of the Mean Squared Error are good indicators in the choice of the appropriate dimensionless numbers. In particular, the strong influence of both vapour quality and mass velocity and their independence suggests avoiding regrouping them in the same dimensionless number. This grouping would result in a large Mean Squared Error since the respective evolution of their exponents would be the same.

This separation is enabled by introducing a vapour only Froude number defined as:

$F r_{v o}=\frac{\frac{G}{\sqrt{\rho_{v}}}}{\sqrt{g\left(\rho_{l}-\rho_{v}\right) d}}=\frac{F r_{v}}{x}$

The vapour and liquid qualities are taken into account by means of the Lockhart-Martinelli parameter. The Lockhart-Martinelli parameter can be expressed as a function of the Fanning friction factors of both phases:

$\chi=\sqrt{\frac{f_{l} \rho_{v}}{f_{v} \rho_{l}}}\left(\frac{1-x}{x}\right)$ 
with $f_{i}$ the Fanning friction factor of phase $i$ (liquid or vapour), $\rho_{i}$ the density of phase $i$, and $x$ the vapour quality.

In practice, the Fanning friction factors are evaluated using Churchill correlation (1975):

$f_{i}=2\left(\left(\frac{8}{R e_{i}}\right)^{12}+\left(A_{i}+B_{i}\right)^{-\frac{3}{2}}\right)^{\frac{1}{12}}$

with $R e_{i}$ the Reynolds number of the phase $i$ (liquid or vapour), $A_{i}$ and $B_{i}$ two constants defined as:

$R e_{i}=\frac{x_{i} G d}{\mu_{i}}$

$A_{i}=\left(2.457 \ln \left(\left(\frac{7}{R e_{i}}\right)^{0.9}+0.27\left(\frac{R_{p}}{d}\right)\right)\right)^{16}$

$B_{i}=\left(\frac{37530}{R e_{i}}\right)^{16}$

where $x_{i}$ is the quality of the phase $i, \mu_{i}$ the viscosity of the phase $i, R_{p}$ is the roughness of the glass tube, which is equal to $10^{-7} \mathrm{~m}$ in the present study.

The diameter exponent is particularly influenced by the mass velocity in the vapour only Froude number, and by the vapour and liquid qualities in the Lockhart-Martinelli parameter. Consequently, the Bond number is considered to decrease the constraint on the diameter exponent, which also enables to take into account surface tension contribution. A correlation respecting symmetry dimension is then obtained using these three dimensionless numbers. The distribution of the data points around the optimization function is represented in figure 9 .

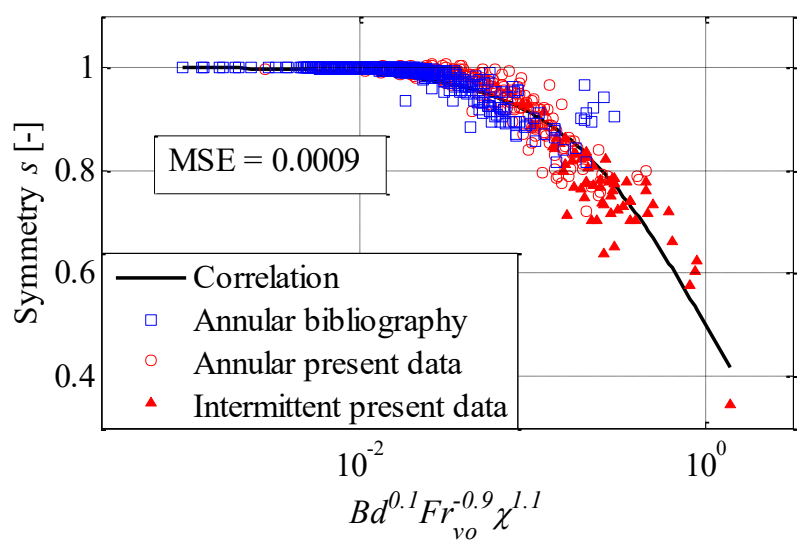

Figure 9: Correlation with the Bond number, the Froude number and the Lockhart-Martinelli parameter This new distribution is characterized by a larger dispersion than the first distribution obtained with the base parameters since the Mean Squared Error is larger in the present case. This larger dispersion is explained by the fact that exponents on the base parameters are constrained by the formulation of the used dimensionless numbers. 
The influence of each exponent on the correlation accuracy is then analyzed with a sensitive study in figure 10. This study enables to justify the equal importance of the three exponents used in the present correlation.

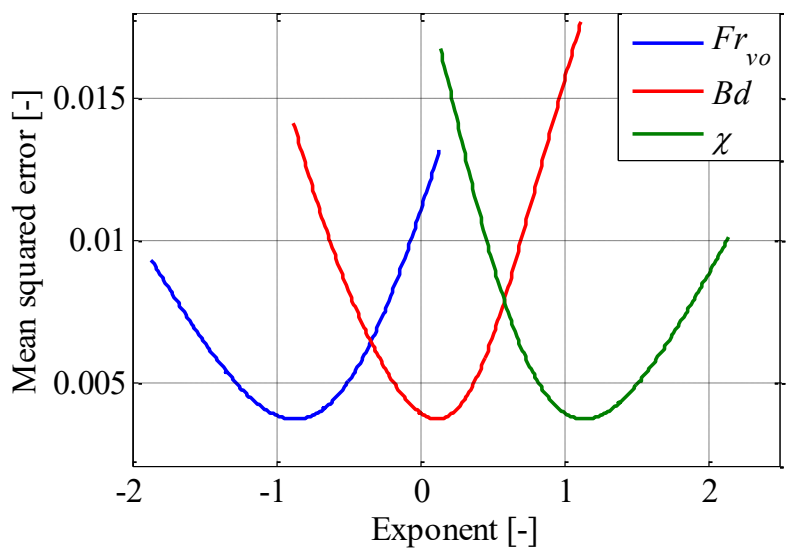

Figure 10: Evolution of the Mean Squared Error with imposed dimensionless number exponents

The three exponents influence the Mean Squared Error with the same intensity, which is close to the intensity previously observed for the vapour quality, the mass velocity and the diameter. Consequently, all exponents present the same importance due to their impact on the Mean Squared Error. These three exponents are equal to $0.1,-0.9$ and 1.1 respectively for the Bond number, the vapour only Froude number and the LockhartMartinelli parameter. The lower value of the Bond number exponent suggests that surface tension affects slightly the asymmetry of the flow in the present range of flow conditions, especially in the range of diameters. Actually, an explanation could be that the surface tension may not clearly affect the symmetry with the proposed definition. The present definition is only affected by the position of vapour core centre. However, surface tension would affect the form of the gas-liquid interface but not the vapour core centre position with the present flow conditions.

Considering the similar importance of the three exponents, a new correlation is then proposed:

$s=\frac{1}{1+B d^{0.1} F r_{v o}^{-0.9} \chi^{1.1}}$

The current optimization enables to have a correlation with a multiplying factor $h$ equal to 1 , which simplifies the function form.

Symmetry then appears to be guided by the relative importance of inertia compared to buoyancy, which is consistent with previous correlations proposed by Hurlburt and Newell (1997), Schubring and Shedd (2009) and Cioncolini and Thome (2013). However, the present method reveals the importance of pressure drops for two-phase flow stratification in horizontal circular mini and macro channels. The lower exponent of the Bond number indicates a lower importance of capillarity in this range of diameters, which is consistent with the known behaviour of mini and macro channels.

The prediction accuracy of the correlation is then analyzed with introduced statistical indicators as shown in figure 11. 


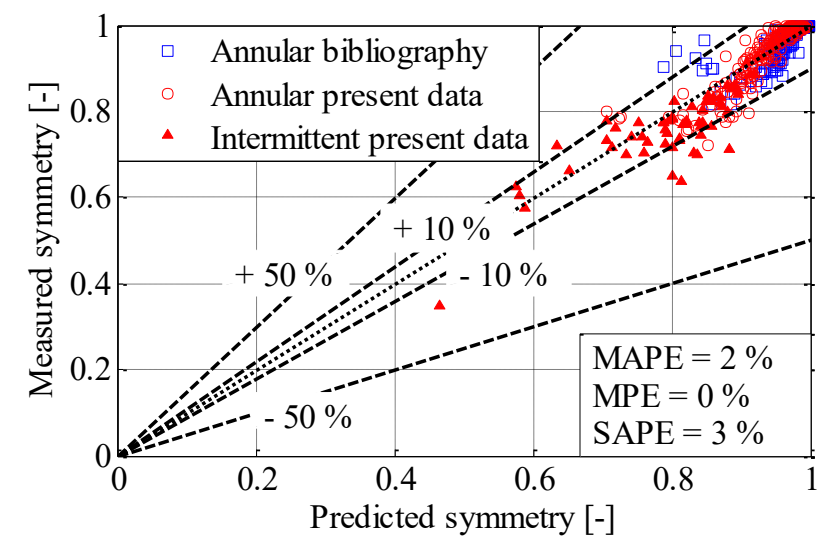

Figure 11: Measured symmetry as a function of predicted symmetry for $B d, \chi$ and $F r_{v o}$ correlation

With this set of exponents, $100 \%$ of the data points are predicted within a $+/-50 \%$ error band whereas $90 \%$ of the database is predicted within $+/-10 \%$ which shows the excellent agreement between the data and the correlation and is consistent with the low values of the MAPE and the SAPE.

The predictions of the correlation are presented in figure 12 for temperature of 40,60 and $80^{\circ} \mathrm{C}$ for the some points of the present database while they are presented for gas superficial velocities of 24,44 and $70 \mathrm{~m} . \mathrm{s}^{-1}$ for other points of the bibliography database. These superficial velocities are calculated with the following formulas:

$U_{s g}=\frac{x G}{\rho_{g}}$

$U_{s l}=\frac{(1-x) G}{\rho_{l}}$

The bibliography data is from Laurinat et al. (1984) study which was considered in the previous correlations of Cioncolini and Thome (2013) and Hurlburt and Newell (1997).

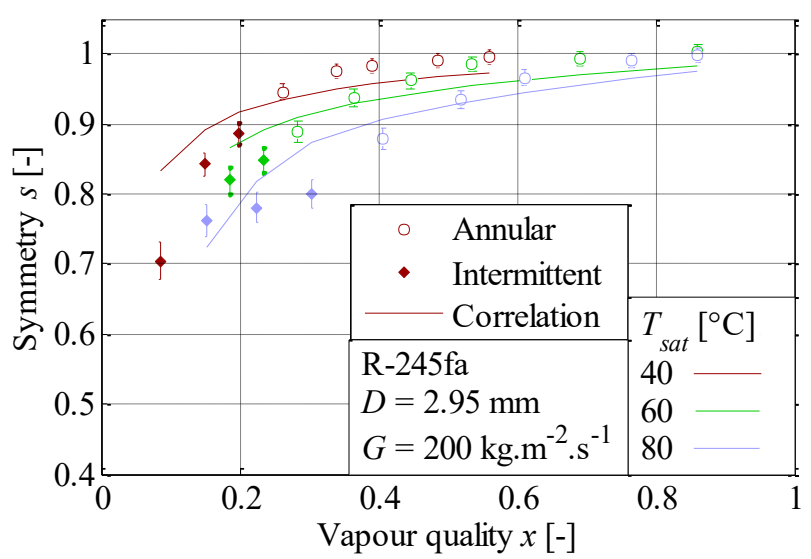

a) Present data 


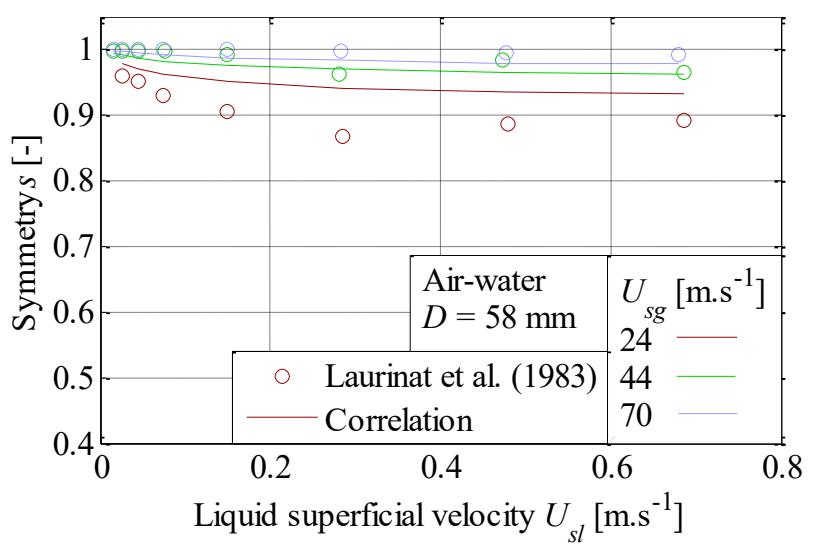

b) Laurinat et al. (1984)

Figure 12: Prediction of the symmetry for a) present data with temperatures of 40,60 and $80^{\circ} \mathrm{C}$ and mass velocity of $200 \mathrm{~kg} \cdot \mathrm{m}^{-2} \cdot \mathrm{s}^{-1}$, b) Laurinat et al. (1984) data with superficial velocities of 24,44 , and $70 \mathrm{~m} \cdot \mathrm{s}^{-1}$

On the present database, the correlation predicts the general trend of the evolution of the symmetry $s$ with vapour quality. A relative error of $+/-10 \%$ is respected for all the points made with annular flows. However, the prediction for the intermittent flows is less accurate with several points whose relative error is higher than $10 \%$. Concerning the Laurinat et al. (1984) data prediction, the most symmetric flows symmetry is better predicted. The dispersion of predictions then appears to be linked to the two considered flows: annular and intermittent.

The evolution of the relative error of the correlation with the vapour quality and the mass velocity for both intermittent and annular flow data points is presented in figure 13. The data points are provided by the present experimental data and the one obtained by Donniacuo et al. (2015), both realized with R-245fa saturated flows. 


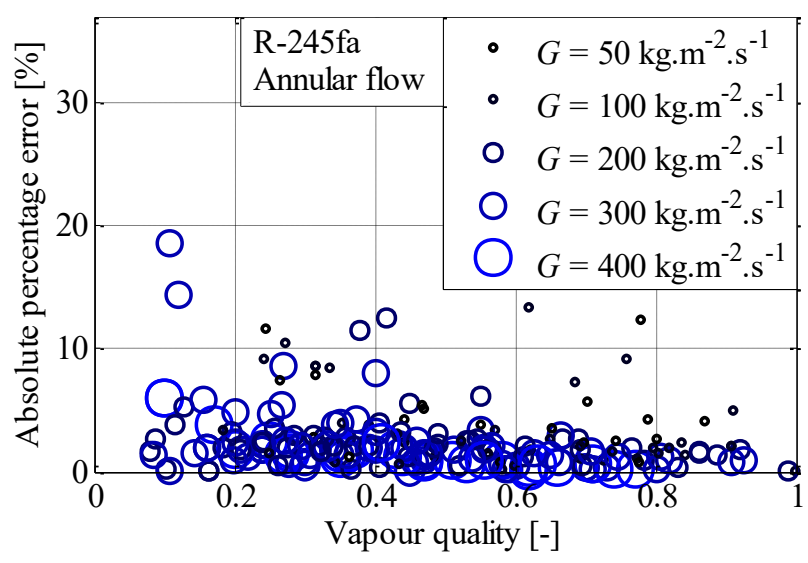

a) Annular data

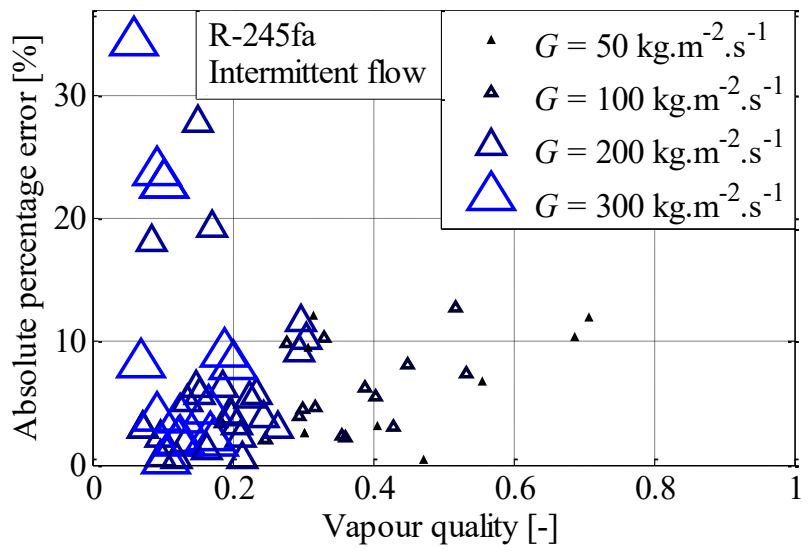

b) Intermittent data

Figure 13: Evolution of the relative error with vapour quality and mass velocity for both a) annular and b) intermittent flow data

Figure 13 shows taht the absolute percentage errors for annular data are lower than those obtained for intermittent data. The maximum errors are obtained for the lowest vapour qualities for both flow patterns. 6 of the 62 intermittent data points are predicted with an absolute percentage error higher than $15 \%$ while they are only 2 for annular data which gathers 208 points. This observation shows the correlation is slightly more accurate to predict annular flows than intermittent flows for the R-245fa database.

This difference of prediction is also shown by comparing the MAPE and the percentage of data captured for both annular and intermittent flows and for the entire database. The various statistical errors are summarized in table 4.

Table 4 : Statistical comparison between annular and intermittent data

\begin{tabular}{cccccccc}
\hline & & & & & \multicolumn{3}{c}{ Percentage captured [\%] } \\
\cline { 6 - 8 } & Pts [-] & MAPE [\%] & MPE [\%] & SAPE [\%] & 5\% errorbar & 10 \% errorbar & 20 \% errorbar \\
\hline Annular & 614 & 1.8 & -0.1 & 2.3 & 92.8 & 98.2 & 100.0 \\
Intermittent & 62 & 7.0 & 0.0 & 6.8 & 53.2 & 82.3 & 96.8 \\
\hline Total & 676 & 2.3 & 0.0 & 3.3 & 89.2 & 96.7 & 99.7 \\
\hline
\end{tabular}


All the statistical indicators presented in table 4 show the slightly higher accuracy of the correlation for the annular flows than for the intermittent flows. These differences of accuracy are probably due to the lower weight of the intermittent flows in the total database (about 10\%). Consequently, the optimization is more affected by annular flows. However, even though the annular flow data is slightly better predicted than intermittent data, all the statistical indicators show the high accuracy of the correlation predictions in the range of experimental conditions.

\section{Conclusions}

A new frame processing was developed to obtain the top and bottom film thicknesses for intermittent flows. It enabled to evaluate two-phase flow asymmetry for more stratified flows than in previous studies. The consideration of a larger range of geometrical configurations led to introduce a new mathematical characterization of asymmetry with the symmetry parameter $s$. This characterization enabled to qualitatively study the evolution of the two-phase flow symmetry $s$ with the Bond number and the vapour Froude number. The shown dependencies led to considerate a method to quantitatively link $s$ to various dimensionless numbers. A bibliographic study was led to consider a large range of conditions, especially for fluid nature and varying diameter. This work enabled to triple the number of data points, gathering 20 studies. A numerical method was introduced to obtain a quantitative correspondence between the symmetry and the flow parameters for all twophase flow patterns. This method took into account the statistical correlations in the database, the uncertainties propagation, and the optimization algorithm robustness to parameters variation to choose appropriate dimensionless numbers. It enabled to develop a new correlation with the Bond number, the vapour only Froude number and the Lockhart-Martinelli parameter. The quantitative dependence of two-phase flow asymmetry with inertia and buoyancy is consistent with past correlations. However, the new correlation considers the dependence of symmetry to the Lockhart-Martinelli parameter, introducing the effect of shear forces. The symmetry dependence on surface tension is also shown to be present even if the Bond number exponent is clearly lower than the two other ones. However, the present database appeared to have a statistical bias by the dependence of diameter to thermophysical parameters. It is necessary to bring other data points to limit this kind of behaviour.

Since this definition of symmetry parameter is linearly linked to vapour core centre position, it is suggested to use a limit value of symmetry parameter $s$ as a criterion of axisymmetry for two-phase flows, considering the limitation of the present database range of diameters.

\section{Acknowledgement}

The bibliographic database was kindly supplied by the Virtual International Research Institute of Two-Phase Flow and Heat Transfer or VIR2AL (http://2phaseflow.org/). We also acknowledge Prof. Schubring and Prof. Shedd for sharing their database. 


\section{References}

Butterworth, D., 1972. Air-water annular flow in a horizontal tube. Proc. Int. Symp. Two-Phase Syst. 235-251.

Charnay, R., Revellin, R., Bonjour, J., 2014. Flow boiling characteristics of R-245fa in a minichannel at medium saturation temperatures. Exp. Therm. Fluid Sci. 59, 184-194.

Churchill, S.W., Chu, H.H.S., 1975. Correlating equations for laminar and turbulent free convection from a horizontal cylinder. Int. J. Heat Mass Transf. 18, 1049-1053.

Cioncolini, A., Thome, J.R., 2013. Liquid film circumferential asymmetry prediction in horizontal annular two-phase flow. Int. J. Multiph. Flow 51, 44-54.

Donniacuo, A., Charnay, R., Mastrullo, R., Mauro, A.W., Revellin, R., 2015. Film thickness measurements for annular flow in minichannels: Description of the optical technique and experimental results. Exp. Therm. Fluid Sci. 69, 73-85.

Fukano, T., Ousaka, A., 1989. Prediction of the circumferential distribution of film thickness in horizontal and near-horizontal gas-liquid annular flows. Int. J. Multiph. Flow 15, 403-419.

Hurlburt, E.T., Newell, T.A., 1997. Prediction of the circumferential film thickness distribution in horizontal annular gas-liquid flow, ACRC Report TR-111.

Jayanti, S., Hewitt, G.F., White, S.P., 1990. Time-dependent behaviour of the liquid film in horizontal annular flow. Int. J. Multiph. Flow 16, 1097-1116.

Laurinat, J.E., Hanratty, T.J., Dallman, J.C., 1984. Pressure drop and film height measurements for annular gas-liquid flow. Int. J. Multiph. Flow 10, 341-356.

Lin, T.F., Block, R.C., Jones, O.C., Lahey, R.T., Murase, M., 1986. Horizontal annular flow measurements using pulsed photon activation and film thickness distribution modelling. Nucl. Eng. Des. 95, 353-363.

Masala, T., Harvel, G., Chang, J.-S., 2007. Separated two-phase flow regime parameter measurement by a high speed ultrasonic pulse-echo system. Rev. Sci. Instrum. 78, 114901.

Ong, C.L., Thome, J.R., 2011. Macro-to-microchannel transition in two-phase flow: Part 1 - Twophase flow patterns and film thickness measurements. Exp. Therm. Fluid Sci. 35, 37-47.

Pletcher, R. H., McManus, H. N., 1965. The fluid dynamics of three dimensional liquid films with free surface shear: A finite difference approach. Presented at the Proceedings of the 9th MidWestern Conference on Mechanics (Developments in Mechanics, pp. 305-318.

Schubring, D., Shedd, T.A., 2009. Critical friction factor modeling of horizontal annular base film thickness. Int. J. Multiph. Flow 35, 389-397.

Williams, L.R., Dykhno, L.A., Hanratty, T.J., 1996. Droplet flux distributions and entrainment in horizontal gas-liquid flows. Int. J. Multiph. Flow 22, 1-18. 